\title{
ABSTRACTS AND COMMENTS FROM THE 2002 BARNA CONFERENCE
}

For those of you who went to the conference in Birmingham you will be familiar with the abstracts printed here. It was not possible to reproduce the whole of the lectures for numerous reasons such as lack of space and in some instances speakers not giving their permission to have their work reproduced. So below are some of the abstracts that speakers kindly submitted for you to read and get a taste of the rich variety of speakers that were at this years conference.

Key note speech: Nurse Anaesthesia: the only appropriate advanced practice of nursing for anaesthetised patients: European Model

\section{Pascal Rod, President IFNA}

Nurse anaesthesia is a very controversial topic in countries where this nursing specialty is not yet developed. The nurse anaesthetist is neither a "small doctor" usurping a medical role and in direct competition with anesthesiologists or a "technician", but a nurse practicing advanced nursing. The nurse anaesthetist is in charge of a patient who is, because of the anaesthetic, temporarily unable to function independently and to perform essential and vital needs. The nursing role is to assist the patient during the anaesthesia maintaining him in a stable physiological state, and providing adapted responses to essential needs. All the technical skills are means that the nurse anaesthetist utilises in responding to the needs of the patient, mostly expressed by clinical signs and/or monitoring data that have to be interpreted. This is the reason why, the nurse anaesthetist has to be prepared and educated beyond the level of general care practice by an appropriate postbasic program of education containing essential topics such as advanced anatomy, physiology, pathophysiology, pharmacology and anaesthetic techniques. This is the European nurse anaesthesia model, based on teamwork and close collaboration between anesthesiologists and nurse anaesthetists.

\section{Recovery of a Cardiac Surgical Patient \\ Dr Justiaan Swanavelder, Consultant} Anaesthetist

Modern cardiac surgery really developed after Gibbon described the first cardiopulmonary bypass using a pump oxygenator in 1953. More than 30000 cardiac operations are now undertaken in the UK each year.

Although the cardiac surgical operating theatre can be intimidating, the principles of anaesthetic care are very similar to those for other procedures. A good understanding of cardiac anatomy and physiology is important. In addition the anaesthetist must feel comfortable to manipulate the cardiovascular system with vaso-active and cardio-active pharmacology.

The pre-operative assessment of the patient's history and medical status, especially the ventricular function is necessary to formulate the haemodynamic goals and anaesthetic management plan. These goals depend on the cardiovascular pathology of the specific patient. Extensive and accurate monitoring is essential throughout the peri-operative period. Apart from the routine anaesthetic monitoring, invasive arterial and central venous pressure determinations are standard of care. The use of a pulmonary artery catheter and/or transoesophageal echocardiography contributes to a better intra-operative assessment of the patient. No anaesthetic drug has been proven best for a patient undergoing a cardiac procedure. It does not matter so much what drugs the anaesthetist use, but how it is used.

During an open-heart operation the functions of the heart and lungs are taken over by the cardiopulmonary bypass (CPB) machine. To initiate this the patient needs to be fully anticoagulated with heparin $(300 \mathrm{U} / \mathrm{kg})$. Although a perfusionist is managing the CPB machine, the anaesthetist is still responsible for the patient during CPB. After the cessation of CPB the effect of heparin is reversed with protamine $(1 \mathrm{mg} / \mathrm{kg}$ for every $100 \mathrm{U} / \mathrm{kg}$ of heparin the patient has received). The 
patient may be haemodynamically unstable during the immediate period after CPB.

The advent of off-pump coronary artery bypass surgery (OPCAB) brought a new dimension to cardiac surgery. This procedure does not make use of CPB and the surgeon operates on the beating heart. The advantages are less manipulation of the ascending aorta and avoidance of the potential side effects of CPB. It is still controversial whether this would make a difference to patient outcome.

Most cardiac patients are transported to ICU while still anaesthetised, intubated and ventilated. Post-operative bleeding, haemodynamic instability, hypothermia and other complications may delay extubation and recovery of the patient. Recent changes in cardiac surgical procedures are focused on reducing costs wherever possible. The most expensive time in the course of cardiac surgery is the day of the operation and the first post-operative day. Fast track cardiac surgery emphasises early extubation and reduced duration of stay in the ICU. Early extubation may be defined as less than 6 hours following surgery. Presently there is scientific evidence that this practice is safe and may lead to reduced ICU stay earlier hospital discharge and reduced costs.

There are many special techniques to optimise conditions for early extubation. The use of short acting anaesthetic and pharmacological agents (e.g. targetcontrolled Propofol infusions, Atracurium or Vecuronium, Fentanyl or Remifentanil, etc.) may decrease the need for postoperative ventilation. The use of the laryngeal mask airway means intubation and extubation can be avoided. The successful placement of a thoracic epidural catheter guarantees superior postoperative pain relief.

Not all cardiac patients need to be managed in an ICU post-operatively. A high dependency surgical recovery unit (HDU) equipped to allow mechanical ventilation and invasive monitoring may allow a faster throughput of selected patients. If a patient is relatively young and fulfil specific criteria (quick operation, short cross-clamp time, warm CBP, good ventricle, etc) the anaesthetist may perform an early extubation before transfer to ICU/HDU.

The anaesthetic of a thoracic patient can be very demanding. Often these are elderly patients with severe respiratory and cardiac impairment. Because of the very painful nature of a thoracotomy these patients often need local analgesic techniques (e.g. thoracic epidural, paravertebral or intercostal blocks etc). Isolation of the lung to be operated on necessitates the use of a double-lumen endotracheal tube or bronchial blocker. A fibre-optic bronchoscope needs to be at hand for confirmation of successful lung isolation. High Frequency Jet Ventilation (HFJV) and the Sanders injector are often used in the thoracic theatre. Majority of times the patient's airway is shared between the thoracic surgeon and the anaesthetist and therefore there need to be good communication in theatre. Postoperatively the thoracic patient is usually extubated in the sitting position immediately after the procedure. They can then be cared for in either an HDU or ICU.

The anaesthesia and recovery of patients for cardio-thoracic procedures is very challenging and proper training and experience of anaesthetic nursing staff/ODPs and recovery staff is of the utmost importance.

\section{Recovery of the cardiac patient in ITU/HDU a nursing perspective}

Lynn Deakin, Clinical Educator and ECMO Specialist

It can be argued that the post-operative recovery of a Cardiac Surgical Patient begins pre-operatively with the identification of risk factors such as smoking and obesity which are likely to impair recovery, and the support for the patient to achieve optimum health possible prior to surgery. The patient's recovery is also influenced heavily by the Anaesthetic given and by the nature and complexity of the surgery undertaken. All of these factors will contribute to the recovery process, influencing whether the patient is fast-tracked back to the ward environment on the day of surgery or has a planned overnight stay in the Cardiac Intensive Care Unit.

This presentation addresses the preoperative preparation of the patient and the impact this has on post-operative recovery, before addressing the post operative care of the patient from leaving the operating theatre until their return to the ward. It considers the initial assessment of the patient, their ventilation, sedation and extubation, cardio-vascular stability, body temperature, renal and neurological status and the inclusion / exclusion criteria for fasttracking a patient to the ward versus an overnight stay in Intensive Care. 


\section{Epidural/Spinal - Applied Physiology and Nursing Care}

Julie Jones and Inge Bateman, Nurse Practitioners in Acute Pain Management

This session is aimed at practitioners working in anaesthetics and Post Anaesthetic Care Units. It will cover applied physiology related to epidural and spinal analgesia followed by required observations and the rationale behind these. Discussion of side effects and problems following spinal/epidural analgesia related to clinical experience will conclude the session.

\section{When things go wrong}

Suzette Woodward, Assistant Director Clinical Governance

This talk aims to provide the listener with an overview of risk management and in particular in relation to the investigation of serious incidents using relevant anaesthetic cases. Aspects covered will be the national agenda; role of the National Patient Safety Agency (NPSA); the proposed national incident reporting system; and the role of the Investigations and Inquiries Unit at the Department of Health (DH). The speaker will draw from experience from her present role, also as a risk manager and in working with both the NPSA and the DH.

\section{Fibreoptic airway management: why bother?}

\section{Dr David I Thomas, Consultant Anaesthetist}

Over recent years the availability and use of fibreoptic equipment in anaesthesia has increased dramatically. This has resulted in increased spending within the Anaesthetic / Operating theatre directorate (a scope alone costs, 8000), but it has also increased the work of staff in theatres in preparing, maintaining and sterilising the equipment.

Is it worth it? Are the anaesthetists just playing, making things artificially difficult and delaying the lists, or are there safety and risk management issues here?

In the presentation I hope to show why anaesthetists have joined the physicians and surgeons in using fibreoptic technology for both diagnosis and as an aid to more safely delivering services. I will summarise the history of the development of the endoscope, outline the role of fibreoptics in anaesthesia, discuss the current use of the fibreoptic laryngoscope, and offer an overview on training for anaesthetists and other theatre staff in this area of airway management. I hope to explore the views of delegates on the subject and seek details of personal experience of involvement with anaesthesia when fibreoptic equipment has been used. Above all, I hope that my presentation will be interesting and fun.

\section{Managing the Diabetic patient within perianaesthetic area}

Sara Da Costa, Nurse Consultant in Diabetes

In my experience, there are many difficulties in ensuring that patients receive surgery with their diabetes control optimised, and their clinical risks reduced. It does not appear to be lack of knowledge, rather of anticipating patients needs, and using current systems to benefit patients. The role of the diabetes team in supporting staff looking after such patients as well patients management is key.

The following key points will be discussed in what I hope will be an interactive session- I am hoping to take away ideas to improve practice as well!

- Acute and chronic complications of diabetes- hypo's, hypers etc

- The physiological effects of surgery on diabetes

- The management of diabetes- the common, and the ideal

- The role of preassessment in reducing risk

- The benefits to patients of early involvement of diabetes teams

- Practical issues- sliding scales, anticipatory education for patients

Modernising Emergency Services in the NHS: Redesigning Care for Patients with Fractured Neck of Femurs

Debbie Johnston, Rachel Jenkins and Mark Radford, CIBA 2 Team, Good Hope Hospital

There are increasing demands placed upon the services offered by the modern NHS, due in part to the technical advances that have been achieved in healthcare. In combating these rising expectations, unique and innovative approaches to practice will form an overall strategy for improving the care delivered to patients.

An increasing proportion of the NHS activity is emergencies, both medical and surgical. Such activity is spread between many specialties, and many professional groups. The process's involved in getting these patients the timely and appropriate care is 


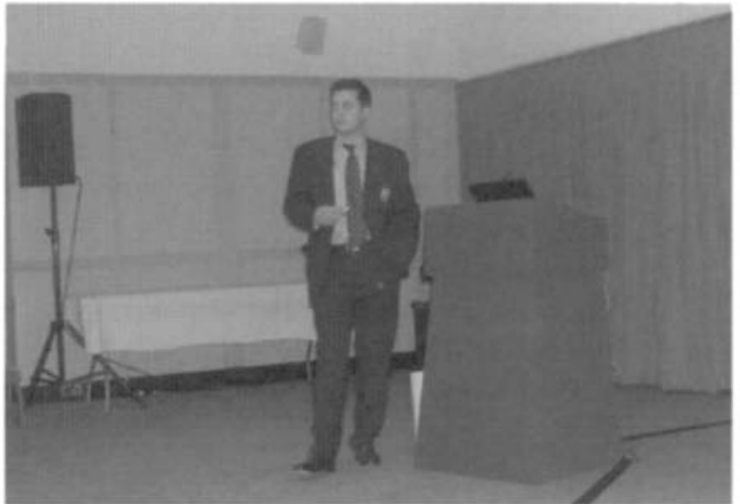

Mark Radford

often complex, and can be disjointed and subject to delay [1][2]. This creates frustrations for patients who require information on their treatment progress. The effect also creates a growing set of problems for clinicians and the organisation.

The CIBA2 Team has managed a project, examining a whole system redesign from the point of injury to discharge. The presentation will describe the practical process of redesign for patients with a fractured neck of femur, looking at the organisational problems faced by these emergency patients. The presentation will also study the process of clinical change and the needs of teams during the redesign process, breaking down barriers and deconstructing the myths within clinical cultures of the NHS. The example used will also demonstrate the use of clinical audit facilitating change in the clinical environment, and the role feedback of positive changes had on team building.

Role development is a key concept in redesigning emergency care, and the presentation will also look at the development and training of the Clinical Nurse Specialist Role in Emergency Surgery and the impact on the care of emergency patients [3], [4]

\section{References}

1. Pearse et al (2001) Organisational features in urgent and emergency surgery. A potential perioperative risk factor, Anaesthesia 56(7): 670-689

2. Crossen-White $H$ et al (1997) The Rise in Emergency Hospital Admission Paper Prepared by Coventry Business School, Coventry University Department of Health Sciences and Clinical Evaluation, University of York. York Health Economics Consortium, University of York

3. Radford $M$ Johnston $P$ Williamson $A$ and Jewkes [2001] A Management of the minor surgical emergency workload by specialist nurse pre-assessment and co-ordination British Journal of Surgery 88[s1] 27
4. Johnston P Williamson A and Radford M [2001] Emergency surgery in the NHS Anaesthesia 56[12] 1203

\section{Neurosurgery Advances: real or apparent?}

Dr James Leggate, Clinical Director Neurosurgery

Over the past 70 years of modern Neurosurgery, many ideas and theories have been tried, dropped and then rediscovered. Ventriculoscopy, Epilepsy surgery and the use of Hypothermia are just a few examples of how some advances have seem to have been made and then lost for many years. Despite the changes of what is in vogue many advances have been realised. The use of the Microscope, Dexamethasone, developments in Neuroanaesthesia and Neurointensive Care are just a few examples of where real progress has been made. What of the future? Will sub-specialisation improve patient outcomes? How will units deal with the ETD (European Time Directive) and still deliver safe neurosurgery. Does the ISAT (International Study of Aneurysm Treatment) trial hold the potential to change forever the role of the Neurosurgeon in the management of patients with Neurovascular diseases? These and other issues will be addressed and discussed with particular reference to Epilepsy surgery, Aneurysm treatment and management of patients with Central Nervous System tumours.

\section{Rapid Weaning off Ventilation in PACU - is it safe?}

Corina Naughton, Research Nurse, Sister in High Dependency Recovery Unit

Introduction: Early reports established the safety and value of a period of intermittent positive pressure ventilation (IPPV) following adult cardiac surgery.1,2 Currently many centers seek to reduce this period significantly. Our goals were to 1) identify clinical factors in our unit associated with delayed extubation. 2) examine the relationship between early extubation and in hospital complications, length of critical care and hospital stay.

Methods: We studied a sequential cohort of 296 unselected adult patients undergoing al cardiac surgery procedures over a threemonth period. Demographic information cardiac risk factors, perioperative and postoperative details were collected. Following univariate analysis, statically significant variables $(\mathrm{P}<0.05)$ were entered into a multiple regression model. 
Results: $39 \%$ of all patients were extubated within 6 hours, 89\% within 24 hours. Delayed extubation ( $>6 \mathrm{hr}$ post surgery) was associated with poor left ventricular, renal and pulmonary function, a high Euroscore, type, duration and urgency of surgery. Modelling using these clinical factors predicted extubation times in $46 \%$ of this cohort.

Early extubation $(<6 \mathrm{hr})$ was not associated with reduced ITU or hospital stay compared to patients who were extubated between 6$24 \mathrm{hrs}$. Complication rates between the two groups were similar (see graph1). Arrthymias, cardiovascular, respiratory and infection were the principle complications associated with a delayed discharge. Patients extubated after 24 hours had significantly longer hospital stay and more post-operative complications.

Fig 1 Complications associated with a delayed extubation in patients extubated within $6 \mathrm{hrs}$ and patients extubated within 6-24 hrs.

Conclusions: In our cohort, patient, surgeryspecific and organisational factors may be influential in determining the duration of postoperative IPPV. Rapid weaning and early extubation was not associated with an increased incidence of post-operative complication. In view of the changing nature of the surgical population, regular reevaluation is useful in reassessing performance.

\section{References:}

1. Cheng D C H [1998] Fast-track cardiac surgery: economic implications in postoperative care. Journal of Cardio thoracic Vascular Anesthesia 12 72-79.

2. Chong J L Pillai R Fisher A et al [1992] Cardiac surgery moving away from intensive care. British Heart Journal 68 430-433.

\section{Neuroanaesthetics - recovery \& critical care patient pathways}

\section{Dr Peter Eadsforth, Consultant Anaesthetist}

Certain characteristics of the brain make it a special case in its regard to being susceptible to injury. A high metabolic rate coupled with a very limited capacity for anaerobic metabolism renders it vulnerable to lack of oxygen supply. Contained in a closed box any brain swelling or haematoma formation can lead to rises in ICP causing brain ischaemia and herniation. The anatomy of the cerebral blood vessels means that interruption of flow down even a small artery can produce infarction of large areas of brain.

A brain that has suffered some form of insult (trauma, surgery, and subarachnoid haemorrhage) is very susceptible to hypoxia, hypotension, hypercarbia, and hyperglycaemia and to the development of swelling or intracranial haematoma formation. Therefore appropriate postoperative monitoring of Neurosurgical patients including Cardio-respiratory function and neurological function is essential in the prevention of secondary brain injury. Accurate recording of observations, together with the recognition of developing problems, can lead to prompt investigation and treatment in the immediate post-operative phase, which may prevent serious morbidity or even death.

\section{Post Anaesthetic recovery in children: A case for specialist training}

Dr George H. Meakin, Senior Lecturer in Paediatric Anaesthesia

Children have special needs reflecting fundamental psychological, anatomical and physiological differences to adults. These needs are best met by having a designated paediatric recovery area, which is child friendly and staffed by nurses trained in the recovery of paediatric patients. In this session the fundamental differences between paediatric and adult patients will be reviewed and their effects on postanaesthesia recovery care explained. There will also be a brief overview of the common post-anaesthetic complications. By the end of the session, delegates should have an understanding of the specialist training requirements of nurses caring for children in the immediate post-anaesthesia period.

\section{Sue McGinty, Senior Sister for Recovery}

Recovery care of adults and the child is the same but different! The same principles of caring for (ABCD) airway, breathing, circulation / conscious level, drips, drains and drugs all apply but there are vast differences in the application of skills and the approach in paediatrics. Reports such as the Allitt Inquiry 1991 HMSO and Children first. Audit Commission Review: A Study of Hospital Service1993 raised issues about where and by whom children should be cared. So do recovery nurses need children's training? Or do they just need "Specialist Paediatric Recovery training" if they have children's theatre lists at their place of work 


\section{Ensuring Valid Consent}

Mike Surkitt-Parr, Risk Complaints and Litigation Manager

A valid consent requires that:

- It is voluntary

- The patient has the full capacity to understand the nature of the procedure

- The patient is not an adult under a mental disability

- The patient is not a child who is incapable of giving consent.

In order to achieve this an understanding of the legal, ethical and professional principles is required.

To support the processes involved in obtaining consent, a policy that is accessible, up to date and applicable to the clinical areas that it relates to is an essential requirement.

It is now largely accepted that obtaining consent is an integral part of a patient's care and treatment, as opposed to the previous view of a one off process on the day of, or prior to, surgery. There is now more acceptance that the whole process of consent is based on the respect for the autonomy of the individual and their right to selfdetermination. This has required all health care professionals to take a more global view of the subject, and in some areas this has led to a re-evaluation of roles. Importantly, nurses are increasingly recognised as having a major contribution. Acknowledgement of the unique contributions of the different members of the team caring for the patient in relation to the processes involved in obtaining a valid consent is vital.

Other vital elements to support this whole process include:

- Educational processes

- Risk Management strategy

- Adequate time and resources allocated to the process

- Communication skills of the professionals involved

Literature/information to support the information giving processes

The Left Handed Option - sponsored by Abbot Laboratories Ltd

Dr Anthony Rubin, Consultant Anaesthetist

There has been a dramatic increase in the use of regional anaesthesia over the last decade as the advantages have become more clearly established. This has been facilitated by the introduction of new safer long acting local anaesthetics such as levobupivacaine and ropivacaine. Using safer drugs, accurate dosing and placing the drugs in the correct anatomical site reduces the risk of accidents with local anaesthetic agents.

Regional anaesthesia may be used alone or combined with sedation or general anaesthesia as part of multimodal anaesthesia, and is often continued into the postoperative period, and occasionally even after discharge form hospital..

This talk will outline the advantages of the newer local anaesthetic agents, and detail some of the trends in the application of regional anaesthetic methods in the care of the surgical patient. The choice between central blocks such as spinals and Epidurals, and peripheral nerve blocks such as brachial plexus or femoral/sciatic will be addressed. The nursing implications with particular emphasis on ensuring safety will be stressed. The effects of better control of postoperative pain, earlier feeding and ambulation on surgical outcome, rehabilitation and earlier discharge from hospital will all be discussed.

\section{Where are we with standards?}

Open forum session led by Pat Smedley

This lecture will focus on the need to create national standards within the perianaesthetic field and present a plan to accomplish this project. A brief overview of government led initiatives to drive quality care within the NHS will be addressed in the first instance. The report on a recent audit to ascertain how standards are being utilized within hospitals around the UK will be given and followed by an analysis of where standards setting fits in to the larger framework of peri-anaesthetic curriculum /competency/ protocol/audit documentation. The main content of the lecture will propose a user-friendly format for standards documentation together with a breakdown of the clinical functions within the perianaesthetic area for which standards are required. Examples of the draft standards written thus far will be given together with proposals for a time frame and means of presentation. The BARNA standards committee is undertaking this work on behalf of the Association members. There will be enough time at the end of the lecture therefore for delegates (members and nonmembers of the association) to raise questions and comments on the proposals put forward. 


\section{Current Trends in Australian Recovery} Nursing

Lyell Brougham, Clinical Nurse ConsultantGeneral Recovery, Australia

Until recently Recovery Units in Australia were very much a poor cousin to other Acute care Units such as Intensive Care Units. Now Australian Recovery Units are slowly establishing themselves as centers of nursing excellence with a defined body of knowledge based on 15 years of planned research and clinical practice development.

This presentation will outline the current trends in Australian Recovery Nursing practice under the headings of Professional, Management, Education and Clinical Practice. Of most interest to Recovery Nurses in the UK will be the trend towards unique educational clinical practice protocols, developed to define and educate Nurses to an expert level of Recovery clinical practice competence. Clinical continuums, flowcharts etc. will be presented for the core Recovery competencies involved in the expert management of the Airway, Hypotension, Pain, PONV and Hypothermia.

Future practice is envisaged to include a greater efficiency of Recovery patient management by the recognition of 2 levels of advanced Recovery nursing practitioners. Whenever the patient management is predicable, these postgraduate-trained nurses will be able to instigate the appropriate patient management, often using the systems developed by Recovery Nurses.

\title{
THE COLD WARS TRILOGY - MONITORING, PREVENTION AND TREATMENT OF PERIOPERATIVE HYPOTHERMIA
}

\author{
BARNAACTAMED Joint Study Day - Birmingham 20th Sept 2002
}

Actamed generously sponsored this free (for delegates) study day in the heart of Birmingham to play out the Cold Wars Trilogy - against the ever-pervasive menace of perioperative hypothermia. The specialist focus on perioperative hypothermia worked well since different aspects of this complex phenomenon were highlighted.

The day kicked off with 'The defence shield under assault'. A timely reminder from Andrew McVicar on the physiological mechanisms of hypothermia - followed logically with an overview of how anaesthesia affects the normal regulation of body temperature from Dr Ratan Alexander.

Stuart Ashworth covered temperature monitoring in 'Know when the enemy strikes' and Andrew Melling gave an indepth account of his research into the adverse effects of hypothermia (pressure sores and pain). Whether it is more economical to warm all patients having already bought the machinery. Or whether to forecast which patients will be more likely to develop hypothermia. This was the interesting question posed by Simon Dixon in his excellent lecture on 'The cost of losing cold wards' Finally Mark Radford gave an expert overview to include the assessment, management, prevention and treatment of hypothermia - a broad remit but one that he handled with skill and humour.

The importance of pre-emptive action to counteract the enemy attack was brought home at this study day. Pre-warming in many cases would be very beneficial and not impossible to organise in the anaesthetic room. The point was also made that even when giving an anaesthetic lasting half an hour warming should be applied to counteract harmful hypothermia. Warming helps the patient in pain and prevents pressure sore formation.

Thanks to Actamed for an imaginative and informative day, and may the force go with you!

Full transcripts of all the above lectures will be published in the February issue of the British Journal of Anaesthetic and Recovery Nursing

BARNA would like to thank ACTAMED for putting on the 'Cold Wars Trilogy' in Birmingham on 20th September. This was a very enjoyable BARNA/ACTAMED collaboration 